\title{
Surgery for Pyogenic Brain Abscess over 30 Years: Evaluation of the Roles of Aspiration and Craniotomy
}

\author{
Yavuz ARAS ${ }^{1}$, Pulat Akın SABANCI ${ }^{1}$, Nail IZGI' ${ }^{1}$, Osman BOYALI' ${ }^{1}$, Onur OZTURK ${ }^{1}$, Aydin AYDOSELI', \\ Achmet ALI ${ }^{2}$, Altay SENCER ${ }^{1}$, Kemal HEPGUL ${ }^{1}$, Faruk UNAL ${ }^{1}$, Orhan BARLAS ${ }^{1}$ \\ ${ }^{1}$ Istanbul University, Istanbul Medical Faculty, Department of Neurosurgery, Istanbul, Turkey \\ ${ }^{2}$ Istanbul University, Istanbul Medical Faculty, Department of Anesthesiology, Istanbul, Turkey
}

\section{ABSTRACT}

AIM: To evaluate the roles of craniotomy and aspiration in the treatment of pyogenic brain abscess throughout 30 years of computerized tomography.

MATERIAL and METHODS: A retrospective study of 224 patients who were surgically treated at Istanbul Medical Faculty, Department of Neurosurgery between 1982 and 2012 was undertaken. The records were analyzed for demographic, clinical and radiological findings, surgical interventions (resection, free-hand aspiration and image-guided aspiration), data regarding abscesses (etiological factors, site, size, number, localization and identified microorganism), corticosteroid and antibiotic usage and complications, and the outcomes were reviewed.

RESULTS: The male-to-female ratio was 2.2 , and the mean age was $26.2 \pm 1.25$ years. The most common presenting symptom was headache $(56.7 \%)$, followed by nausea and vomiting $(28.5 \%)$. Otitis media and mastoiditis were the most common causes of abscess (41.9\%), and 44 patients had multiple abscesses. Aerobic gram-positive microorganisms were the most frequently isolated pathogens in culture (51.5\%). Primary surgical interventions were as follows: craniotomy and resection (38.8\%), free-hand aspiration (49.1\%) and image-guided aspiration (12.1\%). The aspiration-to-resection ratio was $1.36(64 / 47)$ in the first decade, $2.94(50 / 17)$ in the second decade and 1 (23/23) in the last decade. Age, level of consciousness at the time of admission and potent corticosteroid usage were found to be significantly associated with mortality ( $p=0.001, p \leq 0.001$ and $p=0,038$, respectively). The total morbidity and mortality ratios were $4.9 \%$ and $9.8 \%$, respectively. Seizures were more common in patients of craniotomy group ( $p=0.023$ ).

CONCLUSION: Treatment of pyogenic brain abscess remains challenging, despite advances in surgical and imaging technology, and craniotomy retains a significant role in surgical treatment.

KEYWORDS: Pyogenic brain abscess, Computerized tomography, Surgical treatment, Aspiration, Resection

ABBREVIATIONS: CSF: cerebrospinal fluid, CT: computerized tomography, ENT: ear, nose, throat, GOS: Glasgow outcome score, IUIMF: Istanbul University Istanbul Medical Faculty, IV: intravenous, MRI: magnetic resonance imaging, N/V: nausea and vomiting, PBA: pyogenic brain abscess. 


\section{INTRODUCTION}

Pyogenic brain abscess (PBA) is an acute focal cerebral inflammation that is life-threatening if not treated properly $(6,14)$. The condition is challenging to treat, especially in developing countries. Additionally, PBA also constitutes a considerable problem for immunocompromised patients (22). The treatment of a brain abscess involves aspiration of the pus or excision of the abscess, followed by parenteral antibiotic therapy (21). In the last three decades, the diagnosis and outcome of surgical treatment of pyogenic brain abscesses have improved with the emergence of imaging technologies, image-guided stereotaxy, more effective antibiotics and the development of advanced techniques in detecting causative microorganisms $(3,4,10,14,17,19)$.

The choice of surgical procedure has been a subject of debate in recently published studies $(1,6,7,11,17)$. The use of excision by craniotomy as a standard treatment has declined noticeably, and the preferred method is currently surgical aspiration $(1,10,19)$. However, some authors concluded that craniotomy is the preferred surgical method for $\operatorname{PBA}(6,7,16,26)$.

We aimed to analyze the roles of craniotomy and aspiration in the treatment of PBA. In this study, we present a retrospective cohort analysis of our experience with the surgical treatment of PBA in the first 30 years of the computerized tomography (CT) era.

\section{MATERIAL and METHODS}

Hospital records of patients with PBA who were treated surgically in our clinic between 1982 and 2012 were retrospectively analyzed in a cohort study. Patients who were diagnosed as having tuberculosis or fungal abscess were excluded. Each patient's, age, sex, level of consciousness on admission, general physical and neurological examination findings, diagnostic examinations (skull x-ray, ventriculography, electroencephalography, scintigraphy, angiography, CT, magnetic resonance imaging (MRI)), etiological factors, and side, size, number, identified microorganism and localization of the abscesses were recorded.

The level of consciousness at the time of admission was evaluated on a five-grade scale as follows: Grade 0, conscious without deficit; Grade I, conscious with minor deficit; Grade II, drowsy with medium deficit; Grade III, responsive to painful stimuli with severe deficit; Grade IV, coma. Grades 0, I and II at the time of admission were accepted as good, and grades III and IV were categorized as a bad clinical grade in this study (8).

For the treatment variables, antibiotic usage, corticosteroid therapy (dexamethasone, $16 \mathrm{mg}$ IV bolus, 4x4 mg IV/day, minimum 7 days), number and type of surgical interventions and results of each type of surgery, and complications and outcomes were retrospectively reviewed.

In our institution, the preferred principal surgical method is burr hole aspiration unless there is an absolute craniotomy indication, such as increased intracranial pressure caused by a significant mass effect or a traumatic injury that has introduced foreign materials. Craniotomy is more favorable when the PBA is located in the posterior fossa and if there has been a previous neurosurgical operation.

For the follow-up, patients were examined in the outpatient clinic or assessed using telephone interviews. The functional status of patients after treatment and long-term follow-up was evaluated using the Glasgow outcome score (GOS) (6).

Categorical variables were compared using the chi-squared test or Fisher's exact test. Continuous variables within the groups were compared using an independent t-test or ANOVA for parametric data. All statistical analyses were conducted using SPSS v16.0.p $<0.05$ was considered statistically significant.

\section{RESULTS}

During the 30-year period, 224 patients with pyogenic brain abscesses underwent surgery at Istanbul University, Istanbul Medical Faculty (IUIMF), Department of Neurosurgery.

\section{Patient Population}

The patients included 70 females $(31.2 \%)$ and 154 males $(68.8 \%)$, ranging in age from 1 to 75 years $(26.2 \pm 1.25$ years).

\section{Signs and Symptoms}

The patients' level of consciousness at the time of admission was as follows: 185 (82.6\%) patients with grades 0-2, 39 $(17.4 \%)$ patients with grades $3-4$. Headache (54.9\%) was the most common presenting symptom followed by nausea and vomiting (28.6\%). Other less common presenting symptoms were fever, seizure, confusion and limb weakness (Table I). The duration of symptoms ranged from 1-45 days with a mean of 17 days.

\section{Etiological and Predisposing Factors}

Etiological and predisposing factors are summarized in Table II. The most common causes of abscess were otitis media and mastoiditis (21.9\%). Predisposing factors were not identified in $25.4 \%$ of patients.

Eight patients were admitted to the hospital in the last decade with immunodeficiency: two patients had renal transplantation, two patients had Hodgkin's lymphoma, one patient had nonHodgkin's Lymphoma, one patient had autoimmune hepatitis and two patients had acute leukemia. There was no patient with human immunodeficiency virus infection.

\section{Radiology}

The average number of CT scans performed for each patient was three $(2.89 \pm 0.2)$, whereas 94 out of 224 total patients had $\mathrm{MRI}$ examinations, with a total MRI number of 144 (Figure 1AC).

\section{Number, Size and Locations of Abscesses}

In this series, 46 of the patients (18.8\%) had multiple abscesses (Table III). The number of abscesses was between 2 and 12 for 44 patients. The remaining two patients had more than 12 abscesses. 
Table I: Presenting Signs and Symptoms

\begin{tabular}{|c|c|c|c|c|c|}
\hline Symptoms & Number & $\%$ & Signs & Number & $\%$ \\
\hline Headache & 123 & 54.9 & $\begin{array}{l}\text { Focal Neurological Findings (Paresis, Dysphagia, } \\
\qquad \text { Visual Deficiency) }\end{array}$ & 114 & 50.9 \\
\hline N/N & 64 & 28.6 & Neck Rigidity & 22 & 9.8 \\
\hline Fever & 33 & 14.7 & Herniation Findings & 21 & 9.4 \\
\hline Seizure & 49 & 21.9 & Cerebellar Findings & 21 & 9.4 \\
\hline Confusion & 39 & 17.4 & Papilledema & 60 & 26.8 \\
\hline Limb Weakness & 45 & 20.1 & & & \\
\hline
\end{tabular}

N/V: Nausea and vomiting.

Table II: Etiological and Predisposing Factors

\begin{tabular}{lc}
\hline Etiological and Predisposing Factors & No (\%) \\
\hline Local Dissemination & \\
\hline Otitis Media and Mastoiditis & $49(21.9)$ \\
\hline Paranasal Sinus Infection & $12(5.4)$ \\
\hline Head Trauma & $17(7.6)$ \\
\hline Past Surgical Intervention & $24(10.7)$ \\
\hline Congenital Malformations of Skull & $1(0.44)$ \\
\hline Meningitis & $14(6.3)$ \\
\hline Subtotal & $117(52.2)$ \\
\hline Hematogenous Dissemination & $14(6.3)$ \\
\hline Congenital Heart Diseases & $12(5.4)$ \\
\hline Respiratory Pathway Infection & $8(3.6)$ \\
\hline Immune-Deficiency & $16(7.1)$ \\
\hline Others & $50(22.3)$ \\
\hline Subtotal & $57(25.4)$ \\
\hline Unknown Dissemination & 224 \\
\hline Total & \\
\hline
\end{tabular}

The mean diameter of the abscesses measured was $3.51 \pm 0.14$ $\mathrm{cm}$. The locations of the abscesses are shown in Table III.

Abscesses were on the right side in 98 (43.8\%) patients, on the left side in 103 (46\%) patients and on both sides in $23(10.2 \%)$ patients. The most frequent localization was the frontal lobe (25.3\%). Abscesses were located in the thalamus in 10 patients, in the basal ganglia in 2 patients, in the corpus callosum in 3 patients and in the brain stem in 3 patients (Table III). Of these, 9 were single abscesses, whereas the remaining 9 were in multiple-abscess patients.

\section{Previous Surgical Interventions}

Ten patients had received operations for head trauma before undergoing surgery for PBA. In addition to these patients, 44 patients had previous operations; 17 patients had prior neurosurgical operations: 1 patient for an arteriovenous malformation, 1 patient for aneurysm, 3 patients for cranial osteomyelitis, 8 patients for intracranial tumor and 4 patients for intracerebral hemorrhage; 22 patients had previous ear, nose and throat operations: 19 for otitis media, 1 for mastoiditis, 1 for nasopharyngeal carcinoma and 1 patient for nasal polyps; 5 patients had prior ophthalmology operations.

\section{Microbiologic Findings and Antibiotic Treatment}

Growth in culture from abscess material was positive in $38.4 \%$ of the patients. The identified microorganisms are summarized in Table IV. Aerobic gram-positive microorganisms (52.5\%) were the most frequently isolated pathogens. The less common isolated microorganisms were aerobic gram negative (28.3\%), anaerobic gram positive (14.1\%) and anaerobic gram negative $(5.1 \%)$. In 14 patients, when microorganisms could not be isolated from the abscess material, the microorganisms were isolated in blood, cerebrospinal fluid (CSF), pus from the ear or abscess material from the lung. In addition, cultures yielded no growth from 33 patients; however, microorganisms were observed using gram staining.

Cephalosporins were the most commonly used antibiotics, followed by metronidazole and ornidazole, penicillin, chloramphenicol, aminoglycosides in this series. All of the antibiotics were given intravenously. The mean duration of antibiotic treatment was $47.4 \pm 3.04$ days.

\section{Corticosteroid Usage}

Of the 224 patients, 51 did not receive corticosteroid treatment in any phase of their therapies. Of the remaining 173 patients, 77 patients received regular corticosteroid therapy consisting of $16 \mathrm{mg}$ of dexamethasone per day for at least 7 days before being tapered off. The remaining 96 patients did not receive this regimen in terms of dosage and time and were therefore excluded from this analysis. There was no statistically significant difference in terms of admission clinical grades between the corticosteroid-using and non-using groups $(p=0.091)$. However, mortality was higher in patients given corticosteroid therapy $(15.6 \%$ and $3.9 \%, p=0.045)$ (Table V). 


\section{Surgical Procedures}

The primary surgical interventions performed were as follows: craniotomy and resection in 87 patients (38.8\%), free-hand aspiration in 110 patients (49.1\%) and image-guided aspiration in 27 patients $(12.1 \%)$.

The surgical procedures and their distribution across the three decades of the study are shown in Figure 2 and Table VI. A dramatic increase in the number of aspirations is apparent in the first decade. However, in the second decade, although the number of resections remained constant, the number of aspirations decreased. Finally, in the last decade, the resection and aspiration numbers equalized. In the final decade, 23 patients underwent craniotomy. Of these 23 patients, at least 10 patients $(43.4 \%)$ had absolute indications: 2 patients had a traumatic foreign body, 2 had osteomyelitis and 6 had various previous surgical operations.

Table III: Localizations and Mean Diameters of the Abscesses

\begin{tabular}{|c|c|c|c|c|}
\hline Number & $\begin{array}{c}\text { Free } \\
\text { Hand Aspiration }\end{array}$ & $\begin{array}{l}\text { Image Guided } \\
\text { Aspiration }\end{array}$ & Resection & Total \\
\hline Single & 88 & 15 & 75 & 178 \\
\hline Frontal & 18 & 5 & 22 & 45 \\
\hline Temporal & 19 & 1 & 11 & 31 \\
\hline Parietal & 11 & 3 & 10 & 24 \\
\hline Cerebellar & 8 & 0 & 9 & 17 \\
\hline Occipital & 2 & 2 & 5 & 9 \\
\hline Frontoparietal & 27 & 0 & 16 & 43 \\
\hline Basal nuclei/Diencephalon/Mesencephalon & 3 & 4 & 2 & 9 \\
\hline Multiple & 22 & 12 & 12 & 46 \\
\hline Unilateral Supratentorial & 8 & 5 & 3 & 16 \\
\hline Bilateral Supratentorial & 11 & 3 & 4 & 18 \\
\hline Unilateral Infratentorial & 0 & 1 & 2 & 3 \\
\hline \multirow[t]{2}{*}{ Supra and Infratentorial } & 3 & 3 & 3 & 9 \\
\hline & 110 & 27 & 87 & 224 \\
\hline Mean Diameter of Abscess (cm) & $3.99 \pm 0.22$ & $2.49 \pm 0.28$ & $3.40 \pm 0.17$ & $3.53 \pm 0.13$ \\
\hline
\end{tabular}

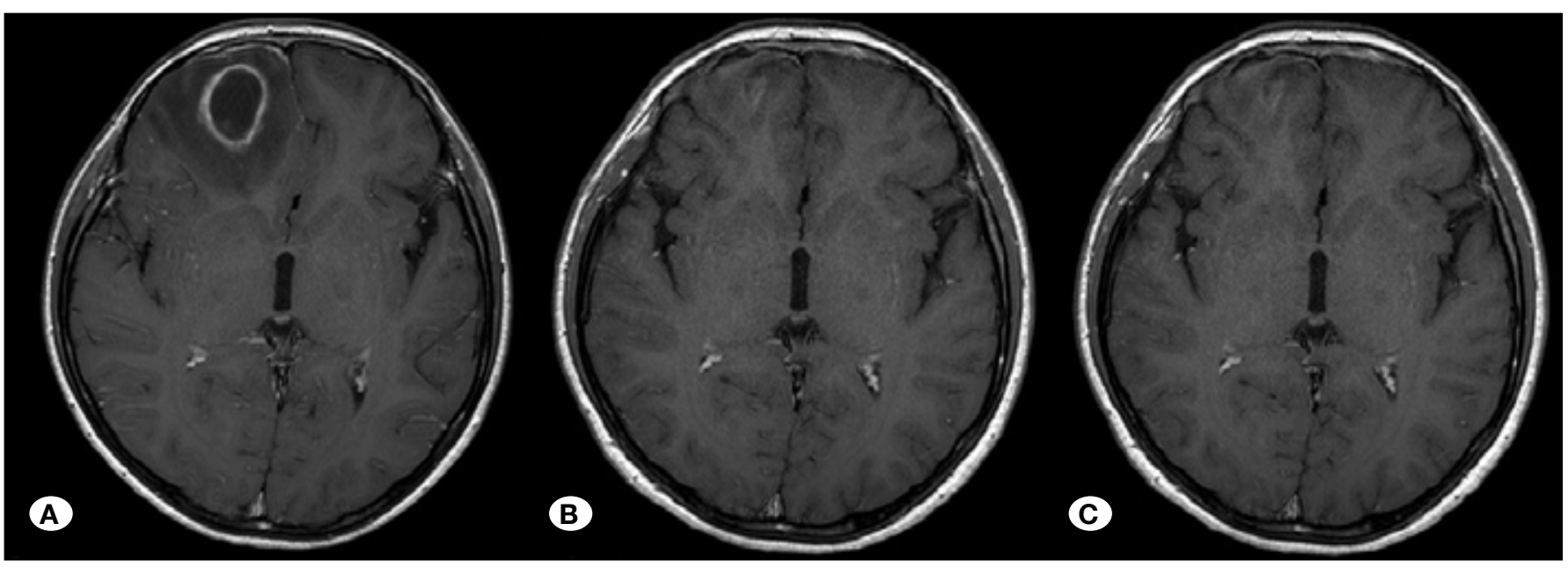

Figure 1: Demonstration of an abscess case. A) A gadolinium-enhanced axialT1-weighted MRI scan showing the characteristic appearance of abscess with peripheral rim enhancement in the right frontal lobe. B, C) Gadolinium-enhanced axial T1-weighted MRI scans obtained 30 and 45 days after aspiration revealing complete resolution of the abscess. 
Multiple aspirations were necessary in 47 patients. The number of aspirations in these patients was 2 to 7 .

Craniotomy and resection were used as a secondary surgical intervention because of recurrence in 19 patients who initially underwent aspiration.

In 25 patients, abscesses were aspirated via frame-based stereotaxy. Multiple abscesses had developed in 12 of these patients. The diameters of the abscesses aspirated by this method ranged between $1 \mathrm{~cm}$ and $5.5 \mathrm{~cm}$ (mean $2.3 \pm 0.27$ $\mathrm{cm})$.

\section{Complications}

The overall surgical complication rate was $25 \%$ (Table VII). In 14 patients, ventricular enlargement occurred following meningitis or ventriculitis in the postoperative period. Eight of these patients required ventriculoperitoneal shunting operations in the follow-up period. Postsurgical de novo neurological deficits were seen in four patients $(1.8 \%)$. In

Table IV: Isolated Pathogens from Abscess Cultures

\begin{tabular}{cc}
\hline & Number (\%) \\
\hline Aerobic Gram (+) & $52(52.5)$ \\
\hline Staphylococcus & $27(27.3)$ \\
\hline Streptococcus & $17(17.2)$ \\
\hline Pneumococcus & 4 \\
\hline Unidentified Gram (+) Bacillus & 4 \\
\hline Aerobic Gram (-) & $28(28.3)$ \\
\hline E. coli & $10(10.1)$ \\
\hline Proteus & $9(9.1)$ \\
\hline Klebsiella & 4 \\
\hline Enterobacter & 3 \\
\hline Acinetobacter & 1 \\
\hline Neisseria & 1 \\
\hline Anaerobic Gram (+) & $14(14.1)$ \\
\hline Anaerobic Streptococcus & $9(9.1)$ \\
\hline Pseudomonas Aeroginosa & 5 \\
\hline Anaerobic Gram (-) & $5(5.1)$ \\
\hline Bacteroides & 5 \\
\hline Total & $99(100)$ \\
\hline
\end{tabular}

nine patients, recurrent abscess formation occurred after the cessation of antibiotic therapy.

\section{Outcomes}

The overall mortality was $9.8 \%$ (22 patients). Among these 22 patients, the causes of death were tentorial or tonsillar herniation at admission in 6 patients, septic shock caused by a secondary infection focus in 2 patients, meningitis and ventriculitis in 2 patients, pulmonary infection in 3 patients, status epilepticus in 1 patient, pulmonary embolism in 2 patients, renal failure in 1 patient, progression of non-Hodgkin's Lymphoma in 1 patient and aneurysmal subarachnoidal hemorrhage in 1 patient. The precise cause of death could not be determined in 3 patients.

Mortality was higher in older patients. The average age was $38.1 \pm 4.68$ years among patients who died and 24.6 \pm 1.25 years in the surviving patients $(p=0.001)$. Mortality was also significantly higher in patients over the age of 18 years $(13.4 \%$ and $5.2 \%, p=0.044$ ).

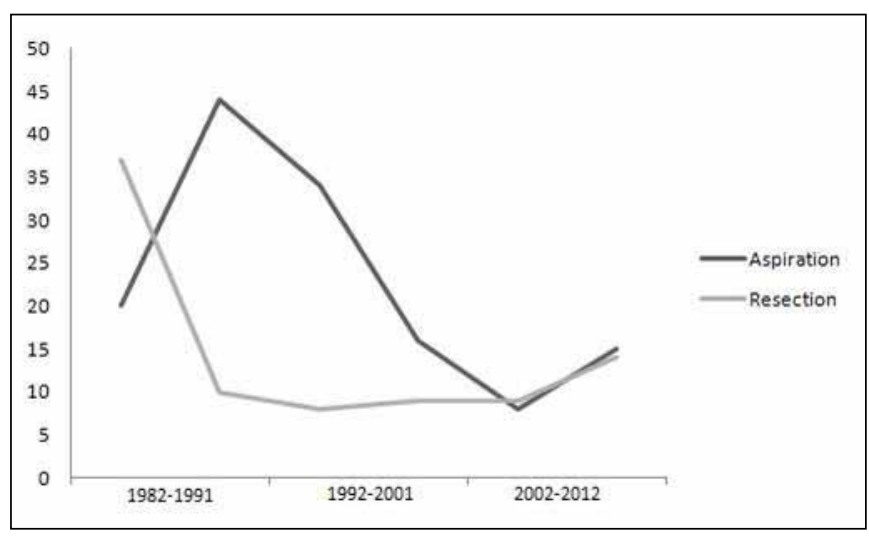

Figure 2: Number of patients according to years.

Table V: Clinical Grades of Patients Who were on Regular Steroid Regimen and Who did not Receive any Steroid

\begin{tabular}{cccl}
\hline Clinical grade & Grade 0-II & Grade III-IV & Total (\%) \\
\hline Steroid use (+) & 54 & 23 & 77 \\
\hline Surviving & 51 & 14 & $65(84.4)$ \\
\hline Died & 3 & 9 & $12(15.6)$ \\
\hline Steroid use (-) & 43 & 8 & 51 \\
\hline Surviving & 43 & 6 & $49(96.1)$ \\
\hline Died & 0 & 2 & $2(3.9)$ \\
\hline
\end{tabular}

Table VI: Number of Procedures among Years

\begin{tabular}{lllll}
\hline & $\mathbf{1 9 8 2 - 9 1}$ & $\mathbf{1 9 9 2 - 2 0 0 1}$ & $\mathbf{2 0 0 2 - 1 2}$ & Total \\
\hline Number of Aspirations & $64(57.6 \%)$ & $50(74.6 \%)$ & $23(50 \%)$ & $137(61.2 \%)$ \\
\hline Number of Resections & $47(42.4 \%)$ & $17(25.4 \%)$ & $23(50 \%)$ & $87(38.8 \%)$ \\
\hline
\end{tabular}


The mortality was $12.6 \%$ in craniotomy patients, $9.1 \%$ in freehand aspiration patients and $3.7 \%$ in image-guided aspiration patients. The mortality in these groups was not statistically different $(p=0.370)$.

Mortality was closely associated with the initial clinical grade and was higher in Grade III-IV patients than in Grade 0-II patients $(38.5 \%$ and $3.8 \%$, respectively, $p \leq 0.001)$. None of the following factors affected mortality: sex, $(9.7 \%$ for women and $10.0 \%$ for men, $p=0.997)$, diameter of abscess at the time of admission $(3.45 \pm 0.15 \mathrm{~cm}$, and $3.98 \pm 0.33 \mathrm{~cm}$ for deceased and surviving patients, respectively, $\mathrm{p}=0.250$ ) and having a single or multiple abscesses $(16.7 \%$, and $8.2 \%, p=0.098)$.

Glasgow outcome scores at discharge are presented in Table VIII. The GOS at discharge was significantly affected by the degree of the patients' clinical grade at the time of admission; $63.8 \%$ of the patients were classified as Grades 0-2 and $30.8 \%$ of patients of Grades 3-4 were at GOS 5 at discharge $(p \geq 0.001)$.

Up to 15 years of follow-up records were available for 109 of the 202 surviving patients. Of the 83 patients with supratentorial abscesses who were followed for more than 1 year, $30.1 \%$
$(42.1 \pm 3.58)$ had seizures. The development of epilepsy was independent of lobar localization $(p=0.330)$. However, the rate of seizures at the follow-up was significantly lower in patients who underwent aspiration (6.8\%) than in those who underwent resection $(18.1 \%),(p=0.023)$.

\section{DISCUSSION}

\section{Abscess Location}

Contiguous spread from otorhinogenic infection was the most common cause of PBA. Additionally, the frontal and temporal lobes and cerebellum are the most common locations of PBA because of their anatomical proximity to the sinuses and ears $(12,18,30)$. In this series, the most common locations of abscesses were the frontal and temporal lobes. The most common predisposing factor was infection of the middle ear and mastoid process (21.9\%). PBA secondary to mastoiditis can be seen in recurrent fashion (25). Our experience of PBA secondary to mastoiditis treatment necessitated a team approach with otolaryngologists. Definitive treatment of mastoiditis is imperative to cure PBA.

Table VII: Complication. Morbidity and Mortality of the Treatment Modalities

\begin{tabular}{lcccc}
\hline & $\begin{array}{c}\text { Free-Hand } \\
\text { Aspiration }\end{array}$ & $\begin{array}{c}\text { Image-Guided } \\
\text { Aspiration }\end{array}$ & Resection & Total \\
\hline Total (\%) & $110(49.1)$ & $27(12.1)$ & $87(38.8)$ & $224(100)$ \\
\hline Complication (\%) & $27(24.5)$ & $3(11.1)$ & $26(29.9)$ & $56(25)$ \\
\hline Local Dissemination of Infection & 4 & 0 & 6 & 10 \\
\hline Hydrocephalus & 7 & 0 & 3 & 10 \\
\hline Intracranial Hematoma & 3 & 1 & 2 & 6 \\
\hline Herniation & 2 & 0 & 5 & 7 \\
\hline CSF Fistula & 2 & 0 & 2 & 4 \\
\hline Systemic Complications & 9 & 2 & 8 & 19 \\
\hline Morbidity (\%) & $3(2.7)$ & $1(3.7)$ & $7(8)$ & $11(4.9)$ \\
\hline Mortality (\%) & $10(9.1)$ & $1(3.7)$ & $11(12.6)$ & $22(9.8)$ \\
\hline
\end{tabular}

CSF: Cerebrospinal fluid.

Table VIII: GOS Scores at Discharge

\begin{tabular}{ccccc}
\hline GOS at discharge & Free-Hand Aspiration & Image-Guided Aspiration & Resection & Total \\
\hline 5 & 67 & 17 & 46 & 130 \\
\hline 4 & 26 & 8 & 26 & 60 \\
\hline 3 & 6 & 0 & 3 & 9 \\
\hline 2 & 1 & 1 & 1 & 3 \\
\hline 1 & 10 & 1 & 11 & 22 \\
\hline
\end{tabular}

GOS: Glasgow Outcome Score. 
The overall incidence of pyogenic brain abscesses in developed nations is increasing with the increased survival of patients with human immunodeficiency virus, the increasing number of transplant patients and the proliferation of immunosuppressive regimens such as antineoplastic chemotherapy $(22,23)$. Furthermore, eight immunodeficient patients were included in this series, all of whom underwent surgery in the last decade. This finding could serve as a warning for the future that more immunodeficient patients with PBA could be encountered.

Computed tomography has enabled the precise detection of multiple abscesses. The patients who had received operations in our clinic for PBA in the pre-CT era were reported by İzi et al. (9). The percentage of multiple abscesses was $5.8 \%(3 / 51)$ in the pre-CT era. In the current study, the ratio of multiple abscesses to the total number of patients studied was $18.8 \%$. The ratio of multiple abscesses has been reported as $13 \%$ $50 \%$ in previous studies $(12,28)$.

\section{Microbiology and Antibiotic Treatment}

The aerobic gram-positive microorganisms, especially Staphylococcus and Streptococcus, were the most common pathogens. These results are compatible with the published literature $(3,18,28)$. Most isolated anaerobic microorganisms in the current study were Peptostreptococcus and Pseudomonas aeruginosa, which was different from previous studies that reported Bacteroides as the most common (3). The successful culture and isolation of microorganisms from the abscess cultures occurred in $38.4 \%$ of the cases in the current study. This ratio was reported as $66.5 \%$ by Yang et al. and $69.9 \%$ by Nathoo et al. $(18,30)$. In 47 patients $(20.9 \%)$, although the abscess cultures were negative, microorganisms were identified by Gram staining or culture from other organs and tissues. The low culture growth rate in the current study was attributed to the use of antibiotics before the cultures were obtained. Negative culture rates were reported to reach $15 \%-30 \%$ in most series $(13,28,30)$, especially in patients for whom antimicrobial therapy was started before operation (6).

New identification methods have been recommended to decrease the negative culture rates. Polymerase chain reaction analysis of $16 \mathrm{~s}$ recombinant DNA and sequencing may identify pathogens to the species level directly from brain abscesses. This approach is rapid and is especially useful in the identification of slow-growing and fastidious organisms $(6,27)$.

In our series, nine patients $(3.6 \%)$ had recurrence after cessation of antibiotic therapy. Of these nine patients, only one had complied with the prescribed six weeks of antibiotic treatment. In the remaining eight patients, early cessation of the treatment, for various reasons, was responsible for the recurrence. In our series, the mean antibiotic usage period was found to be 47 days. Despite six to eight weeks of antibiotic treatment for brain abscesses, the recurrence was reported to be $5 \%-10 \%$ after stopping antibiotic treatment in previous reports $(2,12)$.

\section{Corticosteroid Usage}

In the current study, no significant differences were identified in clinical grades between patients who received corticosteroid therapy and those who did not. However, corticosteroid therapy was associated with a higher mortality rate. Corticosteroid treatment of brain edema caused by brain abscess is controversial (6). Inhibition of the formation of the abscess capsule with dexamethasone was shown in an experimental study by Quartey (20). However, Shroeder (24) found that corticosteroids did not prevent the formation of capsules in abscesses, but it led to a delay in the abscess wall development process.

Mampalam and Rosenblum reported higher mortality rates in patients who received corticosteroid therapy, but these patients' levels of consciousness at the time of admission were worse than those patients who did not receive corticosteroids. Thus, they reported that corticosteroids could be given in patients with progressive neurological deficits and marked brain edema as shown by CT (13). In the current study, no correlation between poor clinical grade at the time of admission (Grades III and IV) and corticosteroid usage was found, in contrast to Mampalam and Rosenblum's report. Mampalam and Rosenblum advocated that corticosteroid therapy should only be used in cases of serious brain edema in PBA patients (13). Gadgil et al. also reported that their utilization of corticosteroid therapy is decided on a case-bycase basis according to the severity of the brain edema (7). Our results also support this suggestion.

\section{Surgical Method}

The treatment of a brain abscess involves aspirating the pus or excising the abscess, followed by parenteral antibiotic therapy (12). However, the choice of surgical method has been the subject of debate (1). The advantages of aspiration are that it is simple, can be used in the cerebritis stage and has less potential morbidity than surgical trauma. In contrast, several reports have advocated excision as the procedure of choice because it is associated with a lower incidence of recurrence and shorter hospitalization $(13,18,22,28)$. Craniotomy and resection with a combination of medical treatment is preferred in the following circumstances: if there is a significant mass effect of the brain abscess, if there are difficulties in the diagnosis, if the abscess is the result of a traumatic injury that has introduced foreign materials and if the lesion is located in the posterior fossa (6). Additionally, some authors prefer to resect single and easily accessible brain abscesses $(7,12,16)$.

Comparison of the number of resection versus aspiration patients throughout this 30 -year period reveals a dramatic increase in the number of aspirations in the first decade of the CT era. However, in the second decade, whereas the number of resections remained stationary, the number of aspirations decreased. Finally, in the last decade, the number of resections and aspirations equalized.

Izgi et al. (9) reported from this institution in the pre-CT era that $94.2 \%$ of PBA patients were treated by craniotomy. The choice of surgical method changed with the introduction of CT. The number of brain abscess patients who were treated by aspiration increased over time (19). Aspiration, repeated as necessary, widely replaced attempts at complete excision. 
In 1999 Barlas et al. reported a more recent series from the same institution and concluded that, "Craniotomy, much advocated in the earlier era when neither antibiotics nor CT was available is now rarely resorted to" (1). Barlas also reported that $\mathrm{CT}$-guided stereotactic aspiration achieved all of the objectives of management, including ascertaining the diagnosis, draining the content of the mass and obtaining pus for accurate diagnosis with low mortality and morbidity (1).

Contrary to Barlas et al.'s report (1), the number of craniotomies increased in the third decade. Of the 23 patients who underwent resection in the third decade, at least 10 patients (43.4\%) had absolute indications; 2 patients had a traumatic foreign body, 2 had osteomyelitis and 6 had various previous surgical operations. The remaining patients underwent craniotomy because of either a diagnostic inaccuracy or the surgeon's preference. Our results also indicate that resection still plays a significant role in the treatment of pyogenic brain abscess.

\section{Outcome}

The most significant determinant of outcome was clinical grade at the time of admission. Of the 39 patients with admission grades III and IV, 24 patients survived, with a mortality rate of $38.5 \%$. In contrast, of the 185 patients with Grades 0-II, only seven patients succumbed, with a mortality rate of only $3.8 \%$. The mortality rates for craniotomy and resection, freehand aspiration and image-guided aspiration were $12.6 \%$, $9.1 \%$ and $3.7 \%$, respectively, in this series $(p=0.370)$. The morbidity rates for craniotomy and resection, free-hand aspiration and image-guided aspiration were $8.1 \%, 2.7 \%$, $3.7 \%(p=0.219)$. These findings are in agreement with other reports $(13,19,28,30)$. Additionally, mortality was significantly higher in patients over the age of eighteen years. Sex, size and location of abscess had no effect on mortality.

\section{Seizure}

Seizures can occur in the process of acute brain abscess formation or following surgical management (6). Furthermore, brain abscesses may cause focal neurological deficits and mental disorders as sequelae (29). Seizure rates of $10 \%-55 \%$ have been reported in the literature $(12,15,29)$.

Focal neurologic deficits were detected in $13.6 \%$ of the surviving 138 patients in the final follow up. Seizures were observed in $29.4 \%$ of patients with supratentorial abscesses who were followed up for more than a year. The type of surgical intervention could affect the probability of seizures. Epilepsy was less frequent in patients who underwent aspiration than in those who underwent resection $(p=0.023)$.

\section{Limitations}

The analysis of results in this case series is limited by the selection biases inherent to a retrospective study. The number of patients treated by image-guided aspiration was relatively small compared to the numbers of craniotomy and freehand aspiration patients. For this reason, it is not possible to compare the mortality and morbidity ratios among these three patient groups.

\section{- CONCLUSION}

This retrospective study of 224 PBAs treated over a period of 30 years has shown that the treatment of brain abscess remains a challenge despite advances in surgical and imaging technology. The most significant determinant of outcome was clinical grade at the time of admission. Corticosteroid therapy was not associated with better outcomes. Comparison of the results of different surgical techniques employed over three decades showed the superiority of aspiration in that postoperative seizures were seen less frequently in this group of patients. The number of aspirations increased dramatically in the first decade of the CT era, decreased in the second decade and equalized with the number of resections in the last decade. Although PBA could be treated successfully by aspiration, a significant number of patients underwent resection for various reasons in the final decade of this series. This study has shown that craniotomy still plays a significant role in the surgical treatment of PBA.

\section{- REFERENCES}

1. Barlas O, Sencer A, Erkan K, Eraksoy H, Sencer S, Bayindir C: Stereotactic surgery in the management of brain abscess. Surg Neurol 52:404-411, 1999

2. Beller AJ, Sahar A, Praiss I: Brain abscess review of 89 cases over a period of 30 years. J Neurol Neurosurg Psychiatry 36:757-768, 1973

3. Brouwer MC, Coutinho JM, van de Beek D: Clinical characteristics and outcome of brain abscess. Neurology 82(9): 806-813, 2014

4. Carpenter J, Stapleton S, Holliman R: Retrospective analysis of 49 cases of brain abscess and review of the literature. Eur $\mathrm{J}$ Clin Microbiol Infect Dis 26:1-11, 2007

5. Chuang MJ, Chang WN, Chang HW, Lin WC, Tsai NW, Hsieh MJ, Wang $\mathrm{HC}$, Lu CH: Predictors and long-term outcome of seizures after bacterial brain abscess. J Neurol Neurosurg Psychiatry 81:913-917, 2010

6. Erdogan E, Cansever T: Pyogenic brain abscess. Neurosurg Focus 24(6):E2, 2008

7. Gadgil N, Patel AJ, Gobinath PS: Open craniotomy for brain abscess: A forgotten experience? Surg Neurol Int 4:34, 2013

8. Garfield J: Management of intracranial abscess: A review of 200 cases. Br Med J 2(5648):7-11, 1969

9. İzgi N, Özden B, Orhon C, Canbolat A, Bilge T: Beyin abseleri. İst Tıp Fak Mec 47: 273-280, 1984, (In Turkish)

10. Landriel F, Ajler P, Hem S, Bendersky D, Goldschmidt E, Garategui L, Vecchi E, Konsol O, Carrizo A: Supratentorial and infratentorial brain abscess: Surgical treatment, complications and outcomes a 10 year single center study. Acta Neurochir 154: 903, 2012

11. Larsen HJ, Astradsson A, Richall H, ErdalJ, Laursen A, Brennum $\mathrm{J}$ : Pyogenic brain abscess, a 15 year survey. BMC Infectious Diseases 12:332, 2012

12. Loftus CM, Osenbach RK, Biller J: Diagnosis and management of brain abscess. In: Wilkins RH, Rengachary SS (eds). Neurosurgery. 2nd ed, Vol: 3, 1996

13. Mampalam TJ, Rosenblum ML: Trends in the management of bacterial brain abscesses: A review of 102 cases over 17 years. Neurosurgery 23:451-457, 1988 
14. Menon S, Bharadwaj R, Chowdhary A, Kaudinya DV, Palande DA: Current epidemiology of intracranial abscesses: A prospective 5 year study. J Med Microbiol 57:1259-1268, 2008

15. Morgan H, Wood MW, Murphey F: Experience with 88 consecutive cases of brain abscess. J Neurosurg 38:698-704, 1973

16. Mut M, Hazer B, Narin F, Akalan N, Ozgen T: Aspiration or capsule excision? Analysis of treatment results for brain abscess at single institute. Turk Neurosurg 19: 36-41, 2009

17. Muzumdar D, Jhawar S, Goel A: Brain abscess: An overview. Int J Surg 9: 136-144, 2011

18. Nathoo N, Nadvi S, Narotam PK, Dellen J: Brain Abscess: Management and outcome analysis of a computed tomography era experience with 973 patients. World Neurosurg 75(5-6):716726, 2011

19. Ratnaike TE, Das S, Gregson BA, Mendelow AD: A review of brain abscess surgical treatment - 78 years: Aspiration versus excision. World Neurosurg 76(5):431-436, 2011

20. Quartey GRC, Johnston JA, Rozdilsky B: Decadron in the treatment of the cerebral abscess: An experimental study. $J$ Neurosurg 45:301-310, 1976

21. Quintana LM: Brain abscess: Aspiration versus excision. Word Neurosurg 76(5): 388-389, 2011

22. Sarmast $A H$, Showkat $H I$, Kirmani AR, Bhat AR, Patloo AM, Ahmad SR, Khan OM: Aspiration versus excision: A single center experience of fourty-seven patients with brain abscess over 10 years. Neurol Med Chir 52: 724-730, 2012
23. Sarmast $A H$, Showkat $H I$, Bhat $A R$, KirmaniAR, Kachroo $M Y$, Mir SF, Alone YR, Khan AA: Analysis and management of brain abscess; a ten year hospital based study. Turk Neurosurg 22(6):682-689, 2012

24. Schroeder KA, McKeever PE, Schabera DR, Hoff JT: Effect of dexamethasone on experimental brain abscess. J Neurosurg 66:264-269, 1987

25. Seven H, Coskun BU, Calis AB, Sayin I, Turgut S: Intracranial abscesses associated with chronic suppurative otitis media. Eur Arch Otorhinolaryngol 262(10):847-851, 2005

26. Su MT, Lan MC, Tsai YD, Lee TC, Lu CH, Chang WN: Multiloculated pyogenic brain abscess: Experience in 25 patients. Neurosurgery 52(5):1075-1079, 2003

27. Tsai JC, Teng LJ, Hsueh PR: Direct detection of bacterial pathogens in brain abscess by polymerase chain reaction amplification and sequencing of $16 \mathrm{~S}$ deoxyribonucleic acid fragments. Neurosurgery 55:1154-1162, 2004

28. Tseng JH, Tseng MY: Brain abscess in 142 patients: Factors influencing outcome and mortality. Surg Neurol 65(6):557-562, 2006

29. Visani P, Schmutzhard E, Trinka E, Pfausler B, Benke T: Subcortical deficit pattern after brain abscess: A neuropsychological study. Eur J Neurol 103(6): 599-603, 2006

30. Yang SY: Brain abscess: A review of 400 cases. J Neurosurg 55:794-799, 1981 